Appl. Set-Valued Anal. Optim. 4 (2022), No. 1, pp. 109-127

Available online at http://asvao.biemdas.com

https://doi.org/10.23952/asvao.4.2022.1.08

\title{
THE CONNECTEDNESS OF WEAKLY AND STRONGLY EFFICIENT SOLUTION SETS OF NONCONVEX VECTOR EQUILIBRIUM PROBLEMS
}

\author{
LAM QUOC ANH ${ }^{1, *}$, NGUYEN THAI ANH ${ }^{1}$, PHAM THANH DUOC ${ }^{2,3}$, \\ LAM THI VAN KHANH ${ }^{1}$, PHAM TRAN ANH THU ${ }^{4}$ \\ ${ }^{1}$ Department of Mathematics, Teacher College, Cantho University, Cantho, Vietnam \\ ${ }^{2}$ Faculty of Mathematics and Computer Science, University of Science, \\ Vietnam National University, Ho Chi Minh City, Vietnam \\ ${ }^{3}$ Faculty of Information Technology, Can Tho University of Technology, Can Tho 900000, Viet Nam \\ ${ }^{4}$ Department of Mathematics, FPT University, Can Tho, Vietnam
}

\begin{abstract}
In this paper, we consider nonconvex vector equilibrium problems, and discuss the properties of their efficient solution sets. First, based on the Hiriart-Urruty oriented distance function, we introduce a new nonlinear scalarization function, and study its continuity properties. Then, we propose various concepts of connectedness for a vector-valued mapping, and discuss their relationships. Finally, we use these concepts to study sufficient conditions of the nonemptyness and connectedness of weakly and strongly efficient solution sets of such problems via the scalarization method and/or conditions related to the triangle inequality.
\end{abstract}

Keywords. Connectedness; Equilibrium problem; Hiriart-Urruty oriented distance function; Scalarization method.

\section{INTRODUCTION}

In recent years, vector equilibrium problems have received much attention of researchers all over the world. These problems unify many important models in vector optimization, such as vector optimization problems, vector variational inequalities, vector complementarity problems, and vector saddle point problems; see, e.g., [1]. Due to the vital roles of vector equilibrium problems, they have been intensively discussed, such as the existence of solutions [2]-[8], the stability and sensitivity analysis [9]-[16], the solution methods [17]-[21], and topological properties of solution sets [22]-[29] and the references therein.

One of the most important topology properties of solution sets is connected. Roughly, this property allows the possibility of moving continuously from one efficient solution to another one. Hence, it plays significant roles in studying algorithm solutions of the problems. Let us present a brief overview of the results devoted to connectedness conditions of efficient solution sets of vector equilibrium problems. In [22], Gong used the linear scalarization method

\footnotetext{
${ }^{*}$ Corresponding author.

E-mail addresses: quocanh@ctu.edu.vn (L.Q. Anh), anhm0721005@gstudent.ctu.edu.vn (N.T. Anh), pt duoc071285@gmail.com (P.T. Duoc), khanhb2000253@student.ctu.edu.vn (L.T.V. Khanh), thupta9@fpt.edu.vn (P.T.A. Thu).
}

Received December 31, 2021; Accepted January 22, 2022.

(C)2022 Applied Set-Valued Analysis and Optimization 
to study the connectedness of Henig weakly efficient solution sets of monotone convex equilibrium problems. In [24], Gong and Yao introduced a positive proper efficient solution set, and proved that this set is dense in the set of efficient solutions of vector equilibrium problems. Then, by using this result and the assumptions related to strong monotonicity and strict convexity, they formulated the connectedness conditions of efficient solution sets of vector equilibrium problems via the linear scalarization. These results and approaches were later extended and improved for set-valued equilibrium problems by Han and Huang [25]. Recently, in [28], Xu and Zhang, based on Hiriart-Urruty oriented distance function, proposed a nonlinear scalarization function for a convex equilibrium problem in Euclidean spaces, and then they employed this function together with properly quasi convex and concave conditions of objective mappings to address the connectedness of efficient solution sets. Very recently, by blending Gerstewitz scalarization function and Hiriart-Urruty oriented distance function, Shao et al. [29] constructed a nonlinear scalarization function and applied it to consider sufficient conditions for the connectedness of efficient solution sets of vector equilibrium problems via free-disposal sets. In the works mentioned above, the convexity conditions of constraint sets and objective mappings are key assumptions, but somehow they are strictly stronger than the connectedness properties of efficient solution sets.

Motivated by this stream of ideas, in this paper, our main aim is to investigate the connectedness conditions of efficient solution sets of nonmonotone and nonconvex vector equilibrium problems. Our contribution is threefold. First, we propose various types of generalized connectedness for vector-valued mappings, and discuss the relationships among them. Second, we provide existence conditions for weakly and strongly efficient solution sets of the reference problems. Third, which is the last, we formulate sufficient conditions for the connectedness of these solution sets without monotonicity and convexity assumptions.

\section{PRELIMINARIES}

Let $\mathbb{X}$ and $\mathbb{Y}$ be normed spaces, and let $\mathscr{C}$ be a pointed, closed, convex, and solid cone in $\mathbb{Y}$. For any $y_{1}, y_{2} \in \mathbb{Y}$, we define the following relation

$$
y_{1} \preccurlyeq \mathscr{C}_{2} \Longleftrightarrow y_{1} \in y_{2}-\mathscr{C} \text {. }
$$

We first recall some notions needed in the sequel.

Definition 2.1. [30, Definition 2.5.1, page 51] A set-valued mapping $G: \mathbb{X} \rightrightarrows \mathbb{Y}$ is said to be

(a) upper semicontinuous (usc) at $x_{0} \in \mathbb{X}$ if, for any neighborhood $\mathscr{V}$ of $G\left(x_{0}\right)$, there exists some neighborhood $\mathscr{U}$ of $x_{0}$ such that $G(\mathscr{U}) \subset \mathscr{V}$;

(b) lower semicontinuous (lsc) at $x_{0} \in \mathbb{X}$ if, for any open subset $\mathscr{V}$ of $\mathbb{Y}$ with $G\left(x_{0}\right) \cap \mathscr{V} \neq \emptyset$, there exists some neighborhood $\mathscr{U}$ of $x_{0}$ such that $G(x) \cap \mathscr{V} \neq \emptyset, \forall x \in \mathscr{U}$;

(c) continuous at $x_{0} \in \mathbb{X}$ if it is both usc and lsc at $x_{0}$.

Remark 2.1. Based on Definition 2.1, we provide the remarks used in what follows.

(a) If the mapping $G$ is not upper semicontinuous at $x_{0} \in \mathbb{X}$, then there exists a neighborhood $\mathscr{V}_{0}$ of $G\left(x_{0}\right)$ such that, for each neighborhood $\mathscr{U}_{n}$ of $x_{0}$, we can find some $x_{n} \in \mathscr{U}_{n}$ satisfying $G\left(x_{n}\right) \cap\left(\mathbb{Y} \backslash \mathscr{V}_{0}\right) \neq \emptyset$, or equivalently there exists some sequence $\left\{x_{n}\right\}$ converging to $x_{0}$ such that, for each $n$, there is $z_{n} \in G\left(x_{n}\right) \backslash \mathscr{V}_{0}$. 
(b) If the mapping $G$ is not lower semincontinuous at $x_{0} \in \mathbb{X}$, then there is some open subset $\mathscr{V}_{0}$ of $\mathbb{Y}$ with $G\left(x_{0}\right) \cap \mathscr{V}_{0} \neq \emptyset$ such that, for each neighborhood $\mathscr{U}_{n}$ of $x_{0}$, there exists some $x_{n} \in \mathscr{U}_{n}$ satisfying $G\left(x_{n}\right) \cap \mathscr{V}_{0}=\emptyset$, or equivalently there exist some $z_{0}$ belonging to $G\left(x_{0}\right)$ and some sequence $\left\{x_{n}\right\}$ converging to $x_{0}$ such that any sequence $\left\{z_{n}\right\}, z_{n} \in G\left(x_{n}\right)$ cannot converge to $z_{0}$.

Definition 2.2. [31, Definition 5.1, page 22] A vector-valued mapping $g: \mathbb{X} \rightarrow \mathbb{Y}$ is said to be

(a) $\mathscr{C}$-lower semicontinuous $\left(\mathscr{C}\right.$-lsc) at $x_{0} \in \mathbb{X}$ if, for any neighborhood $\mathscr{V}$ of $g\left(x_{0}\right)$, there exists some neighborhood $\mathscr{U}$ of $x_{0}$ such that $g(x) \in \mathscr{V}+\mathscr{C}, \forall x \in \mathscr{U}$;

(b) $\mathscr{C}$-upper semicontinuous ( $\mathscr{C}$-usc) at $x_{0} \in \mathbb{X}$ if $-g$ is $\mathscr{C}$-lsc at $x_{0}$;

(c) $\mathscr{C}$-continuous at $x_{0} \in \mathbb{X}$ if it is both $\mathscr{C}$-usc and $\mathscr{C}$-lsc at $x_{0}$.

Remark 2.2. When $\mathbb{Y}=\mathbb{R}$ and $\mathscr{C}=\mathbb{R}_{+}$, the $\mathscr{C}$-lower semicontinuity reduces to the ordinary lower semicontinuity. To be more specify, a function $g$ is said to be lower semicontinuous at $x_{0} \in \mathbb{X}$ if, for every real number $y<g\left(x_{0}\right)$, there exists some neighborhood $\mathscr{U}$ of $x_{0}$ such that $y<g(x)$ for all $x \in \mathscr{U}$, or equivalently for every $\varepsilon>0$, there exists some neighborhood $\mathscr{U}$ of $x_{0}$ such that $g\left(x_{0}\right)-\varepsilon \leq g(x)$ for all $x \in \mathscr{U}$; see, e.g., [32, Definition 2.1] and [33, page 360].

In what follows, we say that a mapping satisfies a given property on $\mathscr{X}$ if it holds one at every point of $\mathscr{X}$. Motivated by [34], we introduce the concepts of the pseudocontinuity for vector-valued mappings. Let $g: \mathbb{X} \rightarrow \mathbb{Y}$ and $\alpha \in \mathbb{Y}$. We consider the $\alpha$-lower level set, denoted by $\operatorname{lev}_{\preccurlyeq \alpha} g:=\{x \in \mathbb{X}: g(x) \preccurlyeq \mathscr{C} \alpha\}$.

Definition 2.3. The vector-valued mapping $g$ is said to be

(a) $\mathscr{C}$-lower pseudocontinuous on $\mathscr{X}$ if lev $\preccurlyeq \alpha g$ is closed for all $\alpha \in g(\mathscr{X})$;

(b) $\mathscr{C}$-upper pseudocontinuous on $\mathscr{X}$ if $-g$ is $\mathscr{C}$-lower pseudocontinuous on $\mathscr{X}$;

(c) $\mathscr{C}$-pseudocontinuous on $\mathscr{X}$ if it is both $\mathscr{C}$-lower pseudocontinuous and $\mathscr{C}$-upper pseudocontinuous on $\mathscr{X}$.

Remark 2.3. It follows from Definition 2.3 that $g$ is $\mathscr{C}$-lower pseudocontinuous at $x_{0} \in \mathbb{X}$ if and only if the set $l e v_{\preccurlyeq g\left(x_{0}\right)} g$ is closed. Therefore, if $g$ is $\mathscr{C}$-lower semicontinuous at $x_{0} \in \mathbb{X}$, then it is also $\mathscr{C}$-lower pseudocontinuous at $x_{0}$. Indeed, for any sequence $\left\{x_{n}\right\} \subset \operatorname{lev}_{\preccurlyeq g\left(x_{0}\right)} g$ converging to $\bar{x}$, we will show that $\bar{x} \in \operatorname{lev}_{\preccurlyeq g\left(x_{0}\right)} g$. Suppose that $\bar{x} \notin \operatorname{lev}_{\preccurlyeq g\left(x_{0}\right)} g$, or equivalently $g(\bar{x}) \in \mathscr{V}:=\mathbb{Y} \backslash\left(g\left(x_{0}\right)-\mathscr{C}\right)$. Due to the openness of the set $\mathscr{V}$ and the lower semicontinuity of $g$ at $x_{0}$, there exists $n_{0} \in \mathbb{N}$ such that $g\left(x_{n}\right) \in \mathscr{V}+\mathscr{C}, \forall n \geq n_{0}$. Consequently, for each $n \geq n_{0}$, there exist $v_{n} \in \mathscr{V}$ and $c_{n} \in \mathscr{C}$ satisfying

$$
g\left(x_{n}\right)=v_{n}+c_{n}
$$

On the other hand, by $x_{n} \in \operatorname{lev}_{\preccurlyeq g\left(x_{0}\right)} g$, we have $g\left(x_{n}\right) \in g\left(x_{0}\right)-\mathscr{C}$. Hence, there is $c_{n}^{\prime} \in \mathscr{C}$ such that $g\left(x_{n}\right)=g\left(x_{0}\right)-c_{n}^{\prime}$. This together with (2.1) would imply that

$$
v_{n}=g\left(x_{0}\right)-c_{n}^{\prime}-c_{n} \in g\left(x_{0}\right)-\mathscr{C},
$$

which is impossible as $v_{n} \in \mathscr{V}=\mathbb{Y} \backslash\left(g\left(x_{0}\right)-\mathscr{C}\right)$.

Now, we provide an example to illustrate that the reverse of above statement is not true in general. 
Example 2.1. Let $\mathbb{X}=\mathbb{Y}=\mathbb{R}, \mathscr{C}=\mathbb{R}_{+}$, and sign $: \mathbb{R} \rightarrow \mathbb{R}$ be defined by

$$
\operatorname{sign}(x)= \begin{cases}1, & \text { if } x>0 \\ 0, & \text { if } x=0 \\ -1, & \text { if } x<0\end{cases}
$$

Then, sign is both $\mathscr{C}$-lower pseudocontinous and $\mathscr{C}$-upper pseudocontinous at $x_{0}=0$, but it is neither $\mathscr{C}$-lower semicontinuous nor $\mathscr{C}$-upper semicontinuous at $x_{0}=0$.

Lemma 2.1. [35, Theorem 4.2.2, page 57] A topological space is compact iff any family of closed sets with the finite intersection property has a nonempty intersection.

We now recall Hiriart-Urruty oriented distance function, introduced in [36] and some its properties.

Definition 2.4. [36, page 83] Hiriart-Urruty oriented distance function $\hbar_{\mathscr{C}}: \mathbb{Y} \rightarrow \mathbb{R}$ is defined by

$$
\hbar_{\mathscr{C}}(y):=d(y, \mathscr{C})-d(y, \mathbb{Y} \backslash \mathscr{C})=\left\{\begin{array}{cl}
d(y, \operatorname{bd} \mathscr{C}), & \text { if } y \notin \mathscr{C}, \\
-d(y, \operatorname{bd} \mathscr{C}), & \text { if } y \in \mathscr{C},
\end{array}\right.
$$

where bd $\mathscr{C}$ is the boundary of $\mathscr{C}$.

Lemma 2.2. [37, Lemma 2.5] Let $y, y_{1}$ and $y_{2}$ be given in $\mathbb{Y}$. Then,

(a) $\hbar_{\mathscr{C}}$ is continuous and convex;

(b) $\hbar_{\mathscr{C}}(y)<0$ if and only if $y \in \operatorname{int} \mathscr{C}$;

(c) $\hbar_{\mathscr{C}}(y)=0$ if and only if $y \in \mathrm{bd} \mathscr{C}$;

(d) $\hbar_{-\mathscr{C}}\left(y_{1}+y_{2}\right) \leq \hbar_{-\mathscr{C}}\left(y_{1}\right)+\hbar_{-\mathscr{C}}\left(y_{2}\right)$;

(e) $y_{1} \preccurlyeq \mathscr{C} y_{2}$ implies $\hbar_{-\mathscr{C}}\left(y_{1}\right) \leq \hbar_{-\mathscr{C}}\left(y_{2}\right)$;

(f) $y_{1} \preccurlyeq$ int $\mathscr{C} y_{2}$ implies $\hbar_{-\mathscr{C}}\left(y_{1}\right)<\hbar_{-\mathscr{C}}\left(y_{2}\right)$.

In the rest of this section, based on Hiriart-Urruty oriented distance function, we introduce a new nonlinear scalarization function, and discuss its properties, which will be used to scalarize vector equilibrium problems. For a vector bifunction $f: \mathbb{X} \times \mathbb{X} \rightarrow \mathbb{Y}$, we consider the bifunction $\eta: \mathbb{X} \times \mathbb{X} \rightarrow \mathbb{R}$ given by

$$
\eta(x, z):=\hbar_{-\mathscr{C}}(f(x, z)), \quad \forall x, z \in \mathbb{X} .
$$

Example 2.2. (a) Let $\mathbb{X}=\mathbb{Y}=\mathbb{R}, \mathscr{C}=\mathbb{R}_{+}$, and $f: \mathbb{R} \times \mathbb{R} \rightarrow \mathbb{R}$. Then, for all $x, z \in \mathbb{R}$,

$$
\eta(x, z)=f(x, z) .
$$

(b) Let $\mathbb{X}=\mathbb{R}, \mathbb{Y}=\mathbb{R}^{2}, \mathscr{X}=\mathscr{C}=\mathbb{R}_{+}^{2}$, and $f: \mathbb{R} \times \mathbb{R} \rightarrow \mathbb{R}^{2}$ be a vector bifunction given by

$$
f(x, z)=\left(f_{1}(x, z), f_{2}(x, z)\right) \text {, }
$$

where $f_{1}, f_{2}: \mathbb{R} \times \mathbb{R} \rightarrow \mathbb{R}$. Then, for all $x, z \in \mathbb{R}$,

$$
\eta(x, z)= \begin{cases}\sqrt{f_{1}^{2}(x, z)+f_{2}^{2}(x, z),} & \text { if } f_{1}(x, z) \geq 0, f_{2}(x, z) \geq 0, \\ f_{2}(x, z), & \text { if } f_{1}(x, z)<0, f_{2}(x, z) \geq 0, \\ f_{1}(x, z), & \text { if } f_{1}(x, z) \geq 0, f_{2}(x, z)<0, \\ \max \left\{f_{1}(x, z), f_{2}(x, z)\right\}, & \text { if } f_{1}(x, z)<0, f_{2}(x, z)<0 .\end{cases}
$$


Next, we study continuity properties of $\eta$, which play important roles in our analysis.

Lemma 2.3. Let $\left(x_{0}, z_{0}\right) \in \mathbb{X} \times \mathbb{X}$ be given. Then,

(a) $\eta$ is upper semicontinuous at $\left(x_{0}, z_{0}\right)$ if $f$ is $\mathscr{C}$-upper semicontinuous at $\left(x_{0}, z_{0}\right)$;

(b) $\eta$ is lower semicontinuous at $\left(x_{0}, z_{0}\right)$ if $f$ is $\mathscr{C}$-lower semicontinuous at $\left(x_{0}, z_{0}\right)$;

(c) $\eta$ is continuous at $\left(x_{0}, z_{0}\right)$ if $f$ is $\mathscr{C}$-continuous at $\left(x_{0}, z_{0}\right)$.

Proof. For the first statement, in view of Remark 2.2, we need to show that, for each $\varepsilon>0$, there exists some neighborhood $\mathscr{U}$ of $\left(x_{0}, z_{0}\right)$ such that $\eta(x, z) \leq \eta\left(x_{0}, z_{0}\right)+\varepsilon, \forall(x, z) \in \mathscr{U}$. Setting $y_{0}:=f\left(x_{0}, z_{0}\right)$, and using Lemma 2.2(a), we can find some neighborhood $\mathscr{V}$ of $y_{0}$ such that

$$
\hbar_{-\mathscr{C}}(y) \leq \hbar_{-\mathscr{C}}\left(y_{0}\right)+\varepsilon, \quad \forall y \in \mathscr{V} .
$$

For the neighborhood $\mathscr{V}$, due to the $\mathscr{C}$-upper semicontinuity of $f$, there is some neighborhood $\mathscr{U}$ of $\left(x_{0}, z_{0}\right)$ such that $f(x, z) \in \mathscr{V}-\mathscr{C}, \forall(x, z) \in \mathscr{U}$. Hence, for each $(x, z)$ belonging to $\mathscr{U}$, we can pick up $y \in \mathscr{V}$ such that $f(x, z) \in y-\mathscr{C}$. By Lemma 2.2(e), we gain

$$
\hbar_{-\mathscr{C}}(f(x, z)) \leq \hbar_{-\mathscr{C}}(y) .
$$

Finally, combining (2.2), (2.3), and (2.4), we obtain $\eta(x, z) \leq \eta\left(x_{0}, z_{0}\right)+\varepsilon, \forall(x, z) \in \mathscr{U}$. Hence, $\eta$ is upper semicontinuous at $\left(x_{0}, z_{0}\right)$. Employing the above techniques, statements (b) and (c) are also verified. This completes the proof.

\section{VARious Kinds of Generalized Connected MaPPing}

In this section, we consider and propose various types of generalized connectedness for vector-valued mappings. We first recall some classical concepts related to the connectedness properties.

Definition 3.1. Let $\mathscr{X}$ be a nonempty subset of $\mathbb{X}$.

(a) [38, page 10]) For each $x_{1}, x_{2} \in \mathbb{X}$, the set $\mathscr{L}_{x_{1}, x_{2}}:=\left\{(1-t) x_{1}+t x_{2}: t \in[0,1]\right\}$ is called a line segment between $x_{1}$ and $x_{2}$. Then, $\mathscr{X}$ is said to be convex if $\mathscr{L}_{x_{1}, x_{2}} \subset \mathscr{X}$ for all $x_{1}, x_{2} \in \mathscr{X}$.

(b) [39, Definition 2.1] For each pair of given points $x_{1}, x_{2} \in \mathbb{X}$, let $\mathscr{A}_{x_{1}, x_{2}}:[0,1] \rightarrow \mathbb{X}$ be a continuous vector-valued mapping such that $\mathscr{A}_{x_{1}, x_{2}}(0)=x_{1}$ and $\mathscr{A}_{x_{1}, x_{2}}(1)=x_{2}$. Then, $\mathscr{A}_{x_{1}, x_{2}}$ is called an arc on $\mathbb{X}$ with endpoints $x_{1}, x_{2}$. The set $\mathscr{X}$ is said to be arcwise connected if, for each pair of points $x_{1}, x_{2}$ in $\mathscr{X}$, there is an $\operatorname{arc} \mathscr{A}_{x_{1}, x_{2}}$ on $\mathscr{X}$.

(c) [40, page 540] A nonempty subset $\mathscr{X}$ of $\mathbb{X}$ is said to be separated if there are two open subsets $\mathscr{U}, \mathscr{V}$ of $\mathbb{X}$ such that $\mathscr{X} \cap \mathscr{U} \neq \emptyset, \mathscr{X} \cap \mathscr{V} \neq \emptyset, \mathscr{U} \cap \mathscr{V}=\emptyset$ and $\mathscr{X} \subset \mathscr{U} \cup \mathscr{V}$. The set $\mathscr{X}$ is said to be connected if it is not separated.

From above definitions, the following statements are easy to check.

$$
\text { convex set } \Longrightarrow \text { arcwise connected set } \Longrightarrow \text { connected set. }
$$

Now we unify and propose general concepts related to convexity properties of mappings.

Definition 3.2. Let $\mathscr{X}$ be a nonempty subset of $\mathbb{X}$. A vector-valued mapping $g: \mathbb{X} \rightarrow \mathbb{Y}$ is said to be 
(a) [31, Definition 6.1, page 29] segmented $\mathscr{C}$-convex $(\mathscr{C}$-convex $)$ on $\mathscr{X}$ if, for all $x_{1}, x_{2} \in \mathscr{X}$, the line segment $\mathscr{L}_{x_{1}, x_{2}}$ is contained in $\mathscr{X}$, and

$$
\forall t \in[0,1], g\left(\mathscr{L}_{x_{1}, x_{2}}(t)\right) \preccurlyeq \mathscr{C}(1-t) g\left(x_{1}\right)+\operatorname{tg}\left(x_{2}\right) ;
$$

(b) [39, Definition 2.2] arcwise connected $\mathscr{C}$-convex on $\mathscr{X}$ if, for all $x_{1}, x_{2} \in \mathscr{X}$, there exists an $\operatorname{arc} \mathscr{A}_{x_{1}, x_{2}}$ on $\mathscr{X}$ such that

$$
\forall t \in[0,1], g\left(\mathscr{A}_{x_{1}, x_{2}}(t)\right) \preccurlyeq \mathscr{C}(1-t) g\left(x_{1}\right)+\operatorname{tg}\left(x_{2}\right) ;
$$

(c) connected $\mathscr{C}$-convex on $\mathscr{X}$ if, for any $x_{1}, x_{2} \in \mathscr{X}$, there exists a connected set $\mathscr{K}_{x_{1}, x_{2}} \subset \mathscr{X}$ containing $x_{1}, x_{2}$ such that $\mathscr{T}_{x_{1}, x_{2}}:=\bigcup_{t \in[0,1]} \mathscr{K}_{x_{1}, x_{2}}(t)$ is connected, where

$$
\mathscr{K}_{x_{1}, x_{2}}(t):=\left\{x \in \mathscr{K}_{x_{1}, x_{2}}: g(x) \preccurlyeq \mathscr{C}(1-t) g\left(x_{1}\right)+\operatorname{tg}\left(x_{2}\right)\right\} .
$$

It follows from the above definitions that every arcwise connected $\mathscr{C}$-convex mapping is connected $\mathscr{C}$-convex. Indeed, if $g$ is arcwise connected $\mathscr{C}_{\mathbb{Y}}$-convex on some subset $\mathscr{X}$ of $\mathbb{X}$, then, for every $x_{1}, x_{2} \in \mathscr{X}$, there exists an arc $\mathscr{A}_{x_{1}, x_{2}}$ on $\mathscr{X}$ such that

$$
\forall t \in[0,1], g\left(\mathscr{A}_{x_{1}, x_{2}}(t)\right) \preccurlyeq \mathscr{C}(1-t) g\left(x_{1}\right)+\operatorname{tg}\left(x_{2}\right) .
$$

By setting $\mathscr{K}_{x_{1}, x_{2}}:=\mathscr{A}_{x_{1}, x_{2}}([0,1])$, we have

$$
\mathscr{T}_{x_{1}, x_{2}}=\bigcup_{t \in[0,1]}\left\{x \in \mathscr{K}_{x_{1}, x_{2}}: g(x) \preccurlyeq \mathscr{C}(1-t) g\left(x_{1}\right)+\operatorname{tg}\left(x_{2}\right)\right\} \subset \mathscr{A}_{x_{1}, x_{2}}([0,1]) .
$$

Moreover, for any $t \in[0,1]$, the vector $\mathscr{A}_{x_{1}, x_{2}}(t)$ is an element of $\mathscr{T}_{x_{1}, x_{2}}$, and consequently the set $\mathscr{T}_{x_{1}, x_{2}}=\mathscr{A}_{x_{1}, x_{2}}([0,1])$ is connected. Therefore, $g$ is connected $\mathscr{C}$-convex on $\mathscr{X}$.

Motivated by [41, Definition 2.1], we have also considered the generalizations of concepts presented in Definition 3.2 as in the followings.

Definition 3.3. Let $\mathscr{X}$ be a nonempty subset of $\mathbb{X}$. A vector-valued mapping $g: \mathbb{X} \rightarrow \mathbb{Y}$ is said to be

(a) [41, Definition 2.1] naturally quasi segmented $\mathscr{C}$-convex (naturally quasi $\mathscr{C}$-convex) on $\mathscr{X}$ if, for all $x_{1}, x_{2} \in \mathscr{X}$, the line segment $\mathscr{L}_{x_{1}, x_{2}}$ is contained in $\mathscr{X}$ and

$$
\forall t \in[0,1], \exists s \in[0,1]: g\left(\mathscr{L}_{x_{1}, x_{2}}(t)\right) \preccurlyeq \mathscr{C}(1-s) g\left(x_{1}\right)+s g\left(x_{2}\right) ;
$$

(b) naturally quasi arcwise connected $\mathscr{C}$-convex on $\mathscr{X}$ if, for all $x_{1}, x_{2} \in \mathscr{X}$, there exists an arc $\mathscr{A}_{x_{1}, x_{2}}$ on $\mathscr{X}$ such that

$$
\forall t \in[0,1], \exists s \in[0,1]: g\left(\mathscr{A}_{x_{1}, x_{2}}(t)\right) \preccurlyeq \mathscr{C}(1-s) g\left(x_{1}\right)+s g\left(x_{2}\right) ;
$$

(c) naturally quasi connected $\mathscr{C}$-convex on $\mathscr{X}$ if, for any $x_{1}, x_{2} \in \mathscr{X}$, there exists a connected subset $\mathscr{K}_{x_{1}, x_{2}} \subset \mathscr{X}$ containing $x_{1}, x_{2}$ such that

$$
\forall x \in \mathscr{K}_{x_{1}, x_{2}}, \exists s \in[0,1]: g(x) \preccurlyeq \mathscr{C}(1-s) g\left(x_{1}\right)+s g\left(x_{2}\right) .
$$

Based on [31, Definition 6.1, page 29], we propose the generalized quasiconvexity concepts of a vector-valued mapping.

Definition 3.4. Let $\mathscr{X}$ be a nonempty subset of $\mathbb{X}$ and an element $\alpha \in \mathbb{Y}$. A vector-valued mapping $g: \mathbb{X} \rightarrow \mathbb{Y}$ is said to be

(a) $\alpha$-lower level quasi segmented $\mathscr{C}$-convex on $\mathscr{X}$ if the set $\operatorname{lev}_{\preccurlyeq \alpha} g \cap \mathscr{X}$ is convex; 
(b) $\alpha$-lower level quasi arcwise connected $\mathscr{C}$-convex on $\mathscr{X}$ if the set $\operatorname{lev}_{\preccurlyeq \alpha} g \cap \mathscr{X}$ is arcwise connected;

(c) $\alpha$-lower level quasi connected $\mathscr{C}$-convex on $\mathscr{X}$ if the set $\operatorname{lev}_{\preccurlyeq \alpha} g \cap \mathscr{X}$ is connected.

Remark 3.1. We denote

$$
\gamma_{1}:=\operatorname{segmented} \mathscr{C}, \quad \gamma_{2}:=\operatorname{arcwise} \text { connected } \mathscr{C}, \quad \gamma_{3}:=\text { connected } \mathscr{C} .
$$

It follows from Definitions 3.2-3.4 that every $\gamma_{i}$-convex (naturally quasi $\gamma_{i}$-convex, $\alpha$-lower level quasi $\gamma_{i}$-convex, respectively) mapping is $\gamma_{j}$-convex (naturally quasi $\gamma_{j}$-convex, $\alpha$-lower level quasi $\gamma_{j}$-convex, respectively) for all $i, j \in\{1,2,3\}$ with $i<j$.

The following examples show that the reverses of Remark 3.1 are not true.

Example 3.1. Let $\mathbb{X}=\mathbb{Y}=\mathbb{R}, \mathscr{X}=\mathbb{R}, \mathscr{C}=\mathbb{R}_{+}$, and $g: \mathbb{R} \rightarrow \mathbb{R}$ be defined by $g(x)=x^{3}$. Then, $g$ is not segmented $\mathbb{R}_{+}$-convex on $\mathbb{R}$. Now, we show that $g$ is arcwise connected $\mathbb{R}_{+}$-convex on $\mathbb{R}$. Taking arbitrarily $x_{1}, x_{2} \in \mathbb{R}$, we set

$$
\mathscr{A}_{x_{1}, x_{2}}(t):=\sqrt[3]{(1-t) x_{1}^{3}+t x_{2}^{3}}, \quad \forall t \in[0,1]
$$

then $\mathscr{A}_{x_{1}, x_{2}}$ is an arc on $\mathbb{R}$. Moreover, we have

$$
g\left(\mathscr{A}_{x_{1}, x_{2}}(t)\right)=(1-t) x_{1}^{3}+t x_{2}^{3}=(1-t) g\left(x_{1}\right)+\operatorname{tg}\left(x_{2}\right) .
$$

Hence, $g$ is arcwise connected $\mathbb{R}_{+}$-convex on $\mathbb{R}$.

Example 3.2. Let $\mathbb{X}=\mathbb{R}^{2}, \mathbb{Y}=\mathbb{R}, \mathscr{X}=\mathbb{R}^{2}, \mathscr{C}=\mathbb{R}_{+}$, and $g: \mathbb{R}^{2} \rightarrow \mathbb{R}$ be defined by $g(\boldsymbol{x})=(x y)^{2}$ for all $\boldsymbol{x}=(x, y) \in \mathbb{R}^{2}$. We next show that $g$ is naturally quasi arcwise connected $\mathbb{R}_{+}$-convex but it is not naturally quasi segmented $\mathbb{R}_{+}$-convex.

$\star g$ is naturally quasi arcwise connected $\mathbb{R}_{+}$-convex on $\mathbb{R}^{2}$ : For each $x_{1}=\left(x_{1}, y_{1}\right)$ and $\boldsymbol{x}_{2}=$ $\left(x_{2}, y_{2}\right)$ in $\mathbb{R}^{2}$, we consider the mapping $\mathscr{A}_{x_{1}, x_{2}}:[0,1] \rightarrow \mathbb{R}^{2}$ defined by

$$
\mathscr{A}_{x_{1}, x_{2}}(t)=\left\{\begin{array}{l}
(1-2 t) x_{1}, \text { if } 0 \leq t \leq 0.5, \\
(2 t-1) x_{2}, \text { if } 0.5<t \leq 1 .
\end{array}\right.
$$

Then, $\mathscr{A}_{\boldsymbol{x}_{1}, \boldsymbol{x}_{2}}$ is an arc on $\mathbb{R}^{2}$. Next, we show that for each $t \in[0,1]$, we can choose $s \in[0,1]$ such that

$$
g\left(\mathscr{A}_{\boldsymbol{x}_{1}, \boldsymbol{x}_{2}}(t)\right) \leq(1-s) g\left(\boldsymbol{x}_{1}\right)+s g\left(\boldsymbol{x}_{2}\right) .
$$

We consider two cases.

Case 1. If $t \in[0,0.5]$, then

$$
g\left(\mathscr{A}_{\boldsymbol{x}_{\mathbf{1}}, \boldsymbol{x}_{\mathbf{2}}}(t)\right)=(1-2 t)^{2}\left(x_{1} y_{1}\right)^{2} \leq\left(x_{1} y_{1}\right)^{2}=g\left(\boldsymbol{x}_{\mathbf{1}}\right) .
$$

Hence (3.1) holds with $s=0$.

Case 2. If $t \in] 0.5,1]$, then

$$
g\left(\mathscr{A}_{\boldsymbol{x}_{1}, \boldsymbol{x}_{2}}(t)\right)=(2 t-1)^{2}\left(x_{2} y_{2}\right)^{2} \leq\left(x_{2} y_{2}\right)^{2}=g\left(\boldsymbol{x}_{2}\right),
$$

and consequently (3.1) is satisfies with $s=1$.

$\star g$ is not naturally quasi segmented $\mathbb{R}_{+}$-convex on $\mathbb{R}^{2}$ : For $\boldsymbol{a}_{1}=(1,5), \boldsymbol{a}_{2}=(5,1)$, and $t=0.5$,

$$
g\left(0.5 \boldsymbol{a}_{\mathbf{1}}+0.5 \boldsymbol{a}_{\mathbf{2}}\right)=g(3,3)=81 \notin 25-\mathbb{R}_{+}=(1-s) g\left(\boldsymbol{a}_{\mathbf{1}}\right)+\operatorname{sg}\left(\boldsymbol{a}_{\mathbf{2}}\right)-\mathbb{R}_{+}, \quad \forall s \in[0,1] .
$$

Therefore, $g$ is not naturally quasi segmented $\mathbb{R}_{+}$-convex on $\mathbb{R}^{2}$. 
Example 3.3. Let $\mathbb{X}=\mathbb{R}^{2}, \mathbb{Y}=\mathbb{R}, \mathscr{X}=\mathbb{R}_{+}^{2}, \mathscr{C}=\mathbb{R}_{+}$, and $g: \mathbb{R}^{2} \rightarrow \mathbb{R}$ be defined by

$$
g(\boldsymbol{x})=g(x, y)= \begin{cases}x^{2}, & \text { if }(x, y) \in \mathscr{V}, \\ y-1, & \text { if }(x, y) \notin \mathscr{V},\end{cases}
$$

where $\mathscr{V}:=\left\{\boldsymbol{x}=(x, y) \in \mathbb{R}^{2}: y=x^{2}\right\} \cap \mathscr{X}$. We will show that $g$ is 3-lower level quasi arcwise connected $\mathscr{C}$-convex but it is not 3 -lower level quasi segmented $\mathscr{C}$-convex.

$\star g$ is 3-lower level quasi arcwise connected $\mathscr{C}$-convex:

For any $\boldsymbol{x}_{1}, \boldsymbol{x}_{2} \in \mathrm{lev}_{\preccurlyeq 3} g \cap \mathscr{X}$, one has $g\left(\boldsymbol{x}_{\mathbf{1}}\right) \leq 3$ and $g\left(\boldsymbol{x}_{\mathbf{2}}\right) \leq 3$. Next, we will find an arc $\mathscr{A}_{x_{1}, x_{2}}$ on $\mathscr{X}$ such that

$$
\forall t \in[0,1], g\left(\mathscr{A}_{x_{1}, x_{2}}(t)\right) \leq 3 .
$$

Assume that $\boldsymbol{x}_{\mathbf{1}}=\left(x_{1}, y_{1}\right)$ and $\boldsymbol{x}_{\mathbf{2}}=\left(x_{2}, y_{2}\right)$. There are three cases to consider. Case 1. If $y_{1} \leq x_{1}^{2}$ and $y_{2} \leq x_{2}^{2}$, then by setting

$$
\mathscr{A}_{\boldsymbol{x}_{1}, \boldsymbol{x}_{2}}([0,1]):=\mathscr{L}_{\boldsymbol{x}_{1},\left(x_{1}, 0\right)} \cup \mathscr{L}_{\left(x_{1}, 0\right),\left(x_{2}, 0\right)} \cup \mathscr{L}_{\left(x_{2}, 0\right), \boldsymbol{x}_{2}},
$$

one has

$$
g\left(\mathscr{A}_{\boldsymbol{x}_{1}, \boldsymbol{x}_{2}}(t)\right)= \begin{cases}g\left(\boldsymbol{x}_{1}\right), & \text { if } t=0, \\ y_{t}-1, & \text { if } 0<t<1, \\ g\left(\boldsymbol{x}_{2}\right), & \text { if } t=1,\end{cases}
$$

where $\mathscr{A}_{x_{1}, x_{2}}(t)=\left(x_{t}, y_{t}\right)$. Consequently,

$$
g\left(\mathscr{A}_{\boldsymbol{x}_{1}, \boldsymbol{x}_{\mathbf{2}}}(t)\right) \leq \max \left\{g\left(\boldsymbol{x}_{\mathbf{1}}\right), g\left(\boldsymbol{x}_{\mathbf{2}}\right), y_{t}-1\right\} \leq \max \left\{g\left(\boldsymbol{x}_{\mathbf{1}}\right), g\left(\boldsymbol{x}_{\mathbf{2}}\right)\right\} \leq 3,
$$

and hence (3.2) holds.

Case 2. If $y_{1}>x_{1}^{2}$ and $y_{2}>x_{2}^{2}$, then

$$
\max \left\{g\left(\boldsymbol{x}_{\mathbf{1}}\right), g\left(\boldsymbol{x}_{\mathbf{2}}\right)\right\}=\max \left\{y_{1}-1, y_{2}-1\right\} \leq 3,
$$

and so $\max \left\{y_{1}, y_{2}\right\} \leq 4$. By setting $\mathscr{A}_{\boldsymbol{x}_{1}, \boldsymbol{x}_{2}}([0,1])=\mathscr{L}_{\boldsymbol{x}_{1}, \boldsymbol{x}_{2}}$, for all $t \in[0,1]$, we achieve $\mathscr{A}_{\boldsymbol{x}_{1}, \boldsymbol{x}_{2}}(t) \notin$ $\mathscr{V}$. Thus,

$$
\left.g\left(\mathscr{A}_{x_{1}, x_{2}}(t)\right)=g\left(x_{t}, y_{t}\right)=y_{t}-1 \leq \max \left\{y_{1}, y_{2}\right)\right\}-1 \leq 3 .
$$

Case 3. If either $y_{1}>x_{1}^{2}, y_{2} \leq x_{2}^{2}$ or $y_{1} \geq x_{1}^{2}, y_{2}<x_{2}^{2}$, then we set

$$
\mathscr{A}_{\boldsymbol{x}_{1}, \boldsymbol{x}_{\mathbf{2}}}([0,1]):=\mathscr{L}_{\boldsymbol{x}_{1},(0,0)} \cup \mathscr{L}_{(0,0),\left(x_{2}, 0\right)} \cup \mathscr{L}_{\left(x_{2}, 0\right), \boldsymbol{x}_{2}} .
$$

It is easy to check that

$$
g\left(\mathscr{A}_{\boldsymbol{x}_{1}, \boldsymbol{x}_{2}}(t)\right) \leq \max \left\{g\left(\boldsymbol{x}_{\mathbf{1}}\right), g\left(\boldsymbol{x}_{\mathbf{2}}\right)\right\} \leq 3
$$

for all $t \in[0,1]$. If either $y_{2}>x_{2}^{2}, y_{1} \leq x_{1}^{2}$ or $y_{2} \geq x_{2}^{2}, y_{1}<x_{1}^{2}$, then statement (3.2) holds with

$$
\mathscr{A}_{\boldsymbol{x}_{1}, \boldsymbol{x}_{2}}([0,1]):=\mathscr{L}_{\boldsymbol{x}_{\mathbf{1}},\left(x_{1}, 0\right)} \cup \mathscr{L}_{\left(x_{1}, 0\right),(0,0)} \cup \mathscr{L}_{(0,0), \boldsymbol{x}_{2}} .
$$

$\star g$ is not 3-lower level quasi segmented $\mathscr{C}$-convex:

For $z_{1}=(0,4), z_{2}=(4,4)$ and $t=0.5$, we have

$$
g\left(z_{1}\right)=g\left(z_{2}\right)=3 \leq 3<4=g\left(0.5 z_{1}+0.5 z_{2}\right) .
$$

Thus, $g$ is not 3 -lower level quasi segmented $\mathscr{C}$-convex.

Example 3.4. Let $\mathbb{X}=\mathbb{R}^{2}, \mathbb{Y}=\mathbb{R}, \mathscr{X}=\mathbb{R}^{2}$, and $\mathscr{C}=\mathbb{R}_{+}$. We consider a subset $\mathscr{V}$ of $\mathbb{R}^{2}$ defined by $\mathscr{V}=\mathscr{V}_{1} \cup \mathscr{V}_{2}$, where

$$
\mathscr{V}_{1}:=\left\{\boldsymbol{x}=\left(x, x^{2}\right) \in \mathbb{R}^{2}:-1 \leq x \leq 0\right\} \text { and } \mathscr{V}_{2}:=\left\{\boldsymbol{x}=(x, y) \in \mathbb{R}^{2}: x>0, y=\sin (1 / x)\right\} .
$$


Then, $\mathscr{V}$ is connected, but it is not arcwise connected. Now, we define the function $g: \mathbb{R}^{2} \rightarrow$ $\mathbb{R}$ given by

$$
g(\boldsymbol{x})= \begin{cases}0, & \text { if } x \in \mathscr{V} \\ 1, & \text { if } x \notin \mathscr{V}\end{cases}
$$

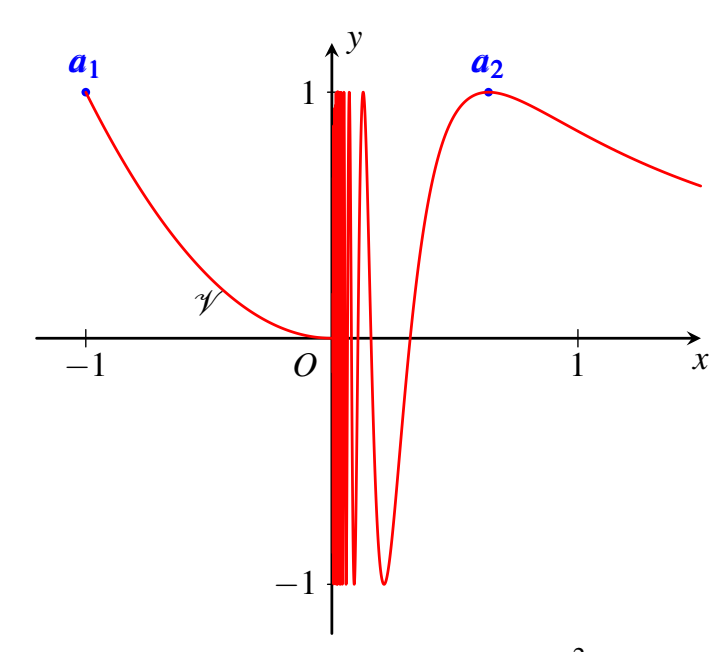

Fig. 1 Displaying of the set $\mathscr{V}$ on $\mathbb{R}^{2}$

We will prove that $g$ is connected $\mathbb{R}_{+}$-convex on $\mathbb{R}^{2}$, but it is not even 0-lower level quasi arcwise connected $\mathbb{R}_{+}$-convex on $\mathbb{R}^{2}$.

$\star g$ is connected $\mathbb{R}_{+}$-convex on $\mathbb{R}^{2}$ : For any $x_{1}, x_{2} \in \mathbb{R}^{2}$, we will choose some connected subset $\mathscr{K}_{\boldsymbol{x}_{1}, \boldsymbol{x}_{2}} \subset \mathscr{X}$ containing $\boldsymbol{x}_{1}, \boldsymbol{x}_{\mathbf{2}}$ such that $\mathscr{T}_{\boldsymbol{x}_{1}, \boldsymbol{x}_{2}}:=\bigcup_{t \in[0,1]} \mathscr{K}_{\boldsymbol{x}_{1}, \boldsymbol{x}_{2}}(t)$ is connected, where

$$
\mathscr{K}_{\boldsymbol{x}_{1}, \boldsymbol{x}_{2}}(t):=\left\{\boldsymbol{x} \in \mathscr{K}_{\boldsymbol{x}_{1}, \boldsymbol{x}_{2}}: g(\boldsymbol{x}) \preccurlyeq \mathscr{C}(1-t) g\left(\boldsymbol{x}_{1}\right)+\operatorname{tg}\left(\boldsymbol{x}_{2}\right)\right\}
$$

There are the following cases to be discussed.

Case 1. If $x_{1}, x_{2} \in \mathscr{V}$, then $(1-t) g\left(x_{1}\right)+\operatorname{tg}\left(x_{2}\right)=0$ for all $t \in[0,1]$. Hence, by setting the set $\mathscr{K}_{x_{1}, x_{2}}:=\mathbb{R}^{2}$, we have

$$
\mathscr{K}_{\boldsymbol{x}_{1}, \boldsymbol{x}_{2}}(t):=\left\{\boldsymbol{x} \in \mathscr{K}_{x_{1}, x_{2}}: g(\boldsymbol{x}) \preccurlyeq \mathscr{C}(1-t) g\left(\boldsymbol{x}_{\mathbf{1}}\right)+\operatorname{tg}\left(\boldsymbol{x}_{2}\right)\right\}=\mathscr{V}, \quad \forall t \in[0,1]
$$

and consequently $\mathscr{T}_{x_{1}, x_{2}}:=\bigcup_{t \in[0,1]} \mathscr{K}_{x_{1}, x_{2}}(t)=\mathscr{V}$ is connected.

Case 2. If either $x_{1} \notin \mathscr{V}$ or $x_{2} \notin \mathscr{V}$, without loss of generality, we assume that $x_{1} \notin \mathscr{V}$. By setting $\mathscr{K}_{x_{1}, x_{2}}:=\mathbb{R}^{2}$, we have

$$
\mathbb{R}^{2} \supset \mathscr{T}_{\boldsymbol{x}_{1}, \boldsymbol{x}_{2}} \supset \mathscr{K}_{\boldsymbol{x}_{1}, \boldsymbol{x}_{2}}(0)=\left\{\boldsymbol{x} \in \mathscr{K}_{\boldsymbol{x}_{1}, \boldsymbol{x}_{2}}: g(\boldsymbol{x}) \preccurlyeq \mathscr{C} 1\right\}=\mathbb{R}^{2} .
$$

So, $\mathscr{T}_{\boldsymbol{x}_{1}, \boldsymbol{x}_{2}}=\mathscr{K}_{\boldsymbol{x}_{1}, \boldsymbol{x}_{2}}(0)=\mathbb{R}^{2}$ is connected.

$\star g$ is not 0 -lower level quasi arcwise connected $\mathbb{R}_{+}$-convex on $\mathbb{R}^{2}$ : For $\boldsymbol{a}_{1}=(-1,1)$ and $\boldsymbol{a}_{2}=(2 / \pi, 1), \boldsymbol{a}_{1} \in \mathscr{V}_{1}$ and $\boldsymbol{a}_{2} \in \mathscr{V}_{2}$. Because $\mathscr{V}$ is not arcwise connected, for any arc $\mathscr{A}_{\boldsymbol{a}_{1}, \boldsymbol{a}_{2}}$ on $\mathbb{R}^{2}$, there is some $\bar{t} \in[0,1]$ such that $\mathscr{A}_{a_{1}, a_{2}}(\bar{t}) \notin \mathscr{V}$, or equivalently

$$
g\left(\mathscr{A}_{\boldsymbol{a}_{1}, a_{2}}(\bar{t})\right)=1>0 .
$$

Therefore, lev $\preccurlyeq 0 g \cap \mathscr{X}$ is not arcwise connected, and so $g$ is not 0-lower level quasi arcwise connected $\mathbb{R}_{+}$-convex on $\mathbb{R}^{2}$. Consequently, $g$ is neither naturally quasi arcwise connected $\mathbb{R}_{+-}$ convex nor arcwise connected $\mathbb{R}_{+}$-convex.

In order to make it easier for understanding the relationships among these concepts, we give the following diagram. 


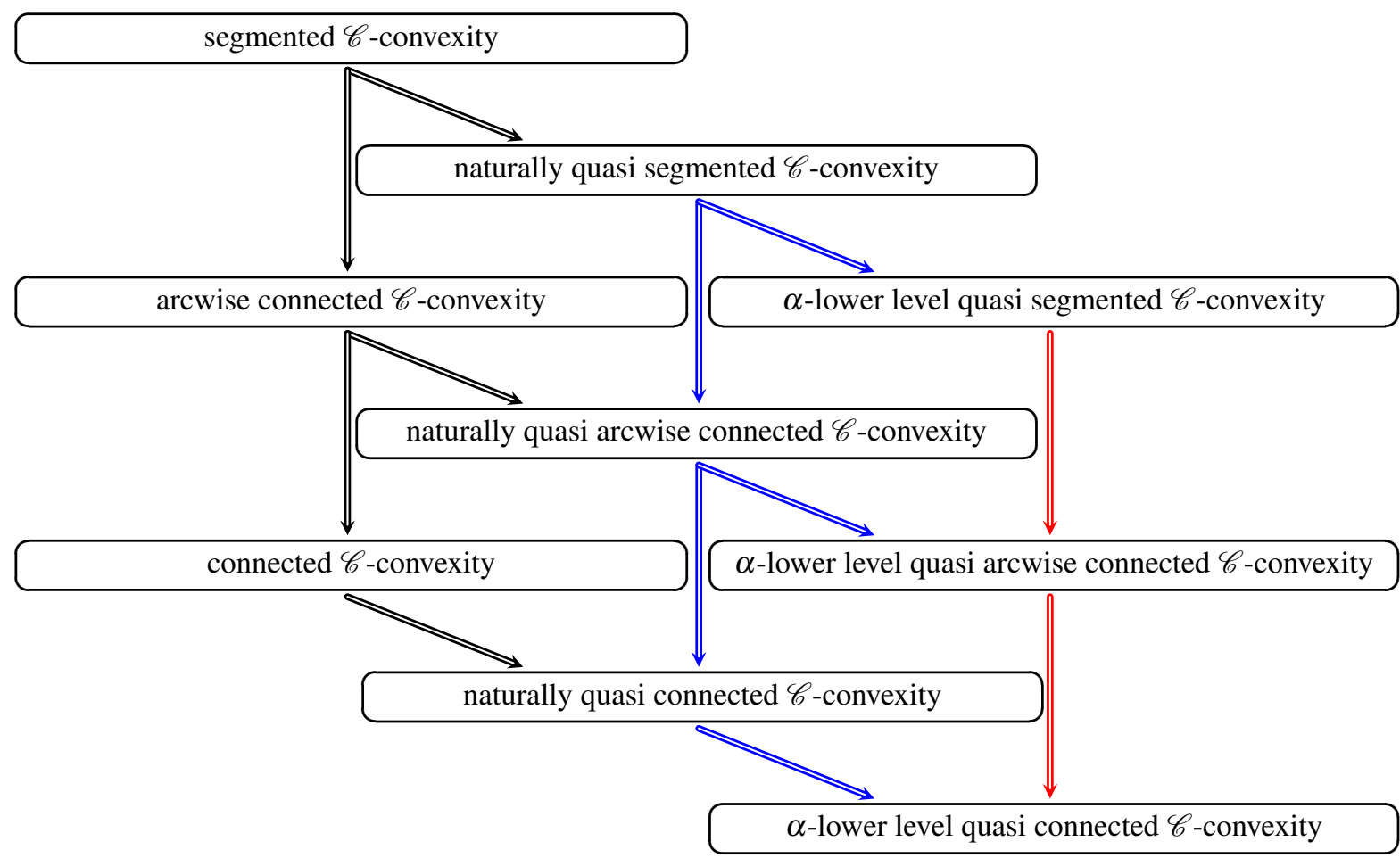

Fig. 2: Relationships among the concepts of generalized convexity

\section{Existence and Connectedness of EfFicient Solution Sets of Nonconvex VECTOR EQUILIBRIUM PROBLEMS}

Let $\mathbb{X}, \mathbb{Y}, \mathscr{C}$ be defined as in Section $2, \mathscr{X}$ be a nonempty subset of $\mathbb{X}$, and let $f: \mathbb{X} \times \mathbb{X} \rightarrow \mathbb{Y}$ be a vector bifunction. We consider the following vector equilibrium problem

(VEP) find $\bar{x} \in \mathscr{X}$ such that

$$
f(\bar{x}, z) \aleph_{\mathscr{C} \backslash\{0\}} 0, \quad \forall z \in \mathscr{X} .
$$

Now we present concepts of efficient solutions of (VEP) as follows.

Definition 4.1. An element $x_{0} \in \mathscr{X}$ is called

(a) a strongly efficient solution of (VEP), written as $x_{0} \in \operatorname{SEff}(\mathscr{X}, f)$ if

$$
0 \preccurlyeq \mathscr{C} f\left(x_{0}, z\right), \quad \forall z \in \mathscr{X} ;
$$

(b) a weakly efficient solution of (VEP), written as $x_{0} \in \operatorname{WEff}(\mathscr{X}, f)$ if

$$
f\left(x_{0}, z\right) \npreceq \text { int } \mathscr{C} 0, \quad \forall z \in \mathscr{X} .
$$

Usually to study the existence of efficient solutions of (VEP), we have to propose assumptions related to convexity of the objective mappings, and hence the constrained sets must be assumed to be convex. However, in practical situations, the convexity conditions often do not hold, and so the class of nonconvex models related to optimization has attracted much attention in recent years. Dealing with nonconvex vector equilibrium problems, the so-called triangle inequality property of the objective bifunction $f$, i.e.,

$$
f(x, z) \leq f(x, y)+f(y, z), \quad \forall x, y, z \in \mathscr{X},
$$


is considered as an efficient tool to investigate the existence of solutions; see e.g., $[3,4]$ and the references therein.

Motivated by above observations, we now introduce a generalized concept of the triangle inequality property of a vector bifunction and apply it to study existence conditions as well as the connectedness property of efficient solution sets of nonconvex vector equilibrium problems.

Definition 4.2. A vector bifunction $f: \mathbb{X} \times \mathbb{X} \rightarrow \mathbb{Y}$ is said to have the $\mathscr{C}$-pseudo triangle inequality property on $\mathscr{X}$ if, for all $x, y, z \in \mathscr{X}$,

$$
[f(y, z) \preccurlyeq \mathscr{C} 0] \Longrightarrow[f(x, z) \preccurlyeq \mathscr{C} f(x, y)] .
$$

Now, we present an example to illustrate the applicability of the class of pseudo triangle inequality functions in a real-life situation.

Example 4.1. Let $\mathbb{G}=\left(\mathscr{S}_{i}, u_{i}\right)_{i \in \mathscr{I}}$ be a weighted potential game, where $\mathscr{I}:=\{1,2, \ldots, n\}$ is a finite set of players, $\mathscr{S}_{i}$ is a strategy set for the $i$-th player, and $u_{i}: \mathscr{S}=\prod_{i \in \mathscr{I}} \mathscr{S}_{j} \rightarrow \mathbb{R}$ is the pay off function for the $i$-th player. Let $w_{i}>0$ for all $i \in \mathscr{I}$. Then, $w=\left(w_{i}\right)_{i \in \mathscr{I}}$ is called a weighted vector; see, e.g., [42]. We set $x=\left(x_{i}, x_{-i}\right)$, where $x_{i} \in \mathscr{S}_{i}$ and $x_{-i} \in \mathscr{S}_{-i}=\prod_{i \neq j \in \mathscr{I}} \mathscr{S}_{j}$. A function $p: \mathscr{S} \rightarrow \mathbb{R}$ is a $w$-potential of $\mathbb{G}$ if, for each player $i$,

$$
u_{i}\left(x_{i}^{\prime}, x_{-i}\right)-u_{i}\left(x_{i}, x_{-i}\right)=w_{i}\left(p\left(x_{i}^{\prime}, x_{-i}\right)-p\left(x_{i}, x_{-i}\right)\right),
$$

for any $x_{i}, x_{i}^{\prime} \in \mathscr{S}_{i}$ and $x_{-i} \in \mathscr{S}_{-i}$. Let $\psi_{i}: \mathscr{S} \times \mathscr{S} \rightarrow \mathbb{R}$ be a real bifunction defined by

$$
\psi_{i}(x, z)=w_{i}(p(z)-p(x)), \quad \forall x, z \in \mathscr{S} .
$$

It is clear that $\psi_{i}$ has $\mathbb{R}_{+}$-pseudo triangle inequality property.

More general, a class of functions with the $\mathbb{R}_{+}$-pseudo triangle inequality property can be built up as follows. Let $\mathscr{X}$ be a nonempty subset of $\mathbb{X}, p: \mathscr{X} \rightarrow \mathbb{R}$ be a real-valued function, and $\mu: \mathbb{R} \rightarrow \mathbb{R}$ be an increasing function such that $\mu(0)=0$. Consider a function $\psi: \mathscr{X} \times \mathscr{X} \rightarrow \mathbb{R}$ defined by $\psi(x, z)=\mu(p(z)-p(x)), \forall x, z \in \mathscr{X}$. Obviously, $\psi$ satisfies the $\mathbb{R}_{+}$-pseudo triangle inequality property.

The following example shows that there are no relationships between the $\mathscr{C}$-pseudo triangle inequality property and the $\mathscr{C}$-convexity in general.

Example 4.2. Let $\mathbb{X}=\mathbb{Y}=\mathbb{R}, \mathscr{X}=\mathbb{R}$, and $\mathscr{C}=\mathbb{R}_{+}$.

(a) Let $f: \mathbb{X} \times \mathbb{X} \rightarrow \mathbb{Y}$ be defined by $f(x, z)=(z-x)^{3}, \forall x, z \in \mathbb{X}$. Then, $f$ satisfies the $\mathbb{R}_{+-}$ pseudo triangle inequality property on $\mathscr{X}$. Indeed, for all $x, y, z \in \mathscr{X}$, if $f(y, z) \preccurlyeq \mathscr{C} 0$, then $z \leq y$, which leads to $(z-x)^{3} \leq(y-x)^{3}$, or equivalently, $f(x, z) \preccurlyeq \mathscr{C} f(x, y)$. However, $f$ is neither $\mathscr{C}$-convex in the first component nor $\mathscr{C}$-convex in the second component on $\mathscr{X}$.

(b) We define the bifunction $h: \mathbb{X} \times \mathbb{X} \rightarrow \mathbb{Y}$ given by $h(x, z)=z^{2}-x, \forall x, z \in \mathbb{X}$. It is clear that $h$ is affine in the first component and convex in the second component on $\mathscr{X}$, but it does not have the $\mathbb{R}_{+}$-pseudo triangle inequality property on $\mathscr{X}$. In fact, for $x=0, y=0.25, z=0.5$, one has

$$
h(y, z)=0 \preccurlyeq \mathscr{C} 0, \quad h(x, z)=0.25 \aleph_{\mathscr{C}} 0.0625=h(x, y) .
$$

Now, we discuss sufficient conditions for the existence of weakly efficient solutions of nonconvex vector equilibrium problems via the nonlinear scalarization function $\eta$ and the pseudo triangle inequality conditions. 
Theorem 4.1. Let $\mathscr{X}$ be a compact subset of $\mathbb{X}$. Assume that

(i) there exists $\bar{x} \in \mathscr{X}$ such that $f(\bar{x}, \cdot)$ is $\mathscr{C}$-lower semicontinuous on $\mathscr{X}$;

(ii) $f$ has int $\mathscr{C}$-pseudo triangle inequality property on $\mathscr{X}$.

Then, $\operatorname{WEff}(\mathscr{X}, f)$ is nonempty. Moreover, if $f$ is $\mathscr{C}$-upper semicontinuous in the first component, then $\operatorname{WEff}(\mathscr{X}, f)$ is compact.

Proof. For the vector $\bar{x} \in \mathscr{X}$ defined in (i), we denote

$$
W(\bar{x}):=\{\bar{z} \in \mathscr{X}: \text { for all } z \in \mathscr{X}, \eta(\bar{x}, z) \geq \eta(\bar{x}, \bar{z})\} .
$$

Since $f(\bar{x}, \cdot)$ is $\mathscr{C}$-lower semicontinuous on $\mathscr{X}$, by Lemma $2.3(b), \eta(\bar{x}, \cdot)$ is lower semicontinuous on $\mathscr{X}$. So, the function $\eta(\bar{x}, \cdot)$ attains the minimal values over the compact subset $\mathscr{X}$, namely, $W(\bar{x})$ is nonempty. Taking arbitrarily $\bar{z} \in W(\bar{x})$, one has

$$
\eta(\bar{x}, z) \geq \eta(\bar{x}, \bar{z})
$$

for all $z \in \mathscr{X}$. If $\bar{z} \notin \operatorname{WEff}(\mathscr{X}, f)$, then we can find $\hat{z} \in \mathscr{X}$ such that $f(\bar{z}, \hat{z}) \preccurlyeq$ int $\mathscr{C} 0$. Combining this with (ii), one has $f(\bar{x}, \hat{z}) \preccurlyeq \operatorname{int} \mathscr{C} f(\bar{x}, \bar{z})$, which together with Lemma 2.2(f) implies that $\eta(\bar{x}, \hat{z})<\eta(\bar{x}, \bar{z})$. This contradicts (4.2). Hence $\bar{z} \in \operatorname{WEff}(\mathscr{X}, f)$. Therefore, $\operatorname{WEff}(\mathscr{X}, f)$ is nonempty.

Furthermore, if $f$ is $\mathscr{C}$-upper semicontinuous in the first component, we prove that $\operatorname{WEff}(\mathscr{X}, f)$ is compact. Let $\left\{x_{n}\right\} \subset \operatorname{WEff}(\mathscr{X}, f)$ be a given sequence converging to some $x_{0}$, we will show that $x_{0}$ belongs to $\operatorname{WEff}(\mathscr{X}, f)$. Since $x_{n} \in \operatorname{WEff}(\mathscr{X}, f)$, one has $f\left(x_{n}, z\right) \notin-\operatorname{int} \mathscr{C}$ for all $z \in \mathscr{X}$. Then, by Lemma 2.2(b), we have

$$
\eta\left(x_{n}, z\right) \geq 0, \quad \forall n \in \mathbb{N}, \forall z \in \mathscr{X} .
$$

Because of the $\mathscr{C}$-upper semicontinuity in the first component of $f$, Lemma 2.3(a) derives that, for each $z \in \mathscr{X}$, the function $\eta(\cdot, z)$ is upper semicontinuous. Due to (4.3), we have $\eta\left(x_{0}, z\right) \geq 0$, $\forall z \in \mathscr{X}$. Applying Lemma 2.2(b), we obtain $f\left(x_{0}, z\right) \notin-$ int $\mathscr{C}$ for all $z \in \mathscr{X}$, or equivalently $x_{0}$ belongs to $\operatorname{WEff}(\mathscr{X}, f)$. Hence $\operatorname{WEff}(\mathscr{X}, f)$ is a compact set as it is a closed subset of the compact set $\mathscr{X}$.

Remark 4.1. In the theorem above, we use the pseudo triangle inequality property to establish the existence conditions for weakly efficient solutions of the vector equilibrium problems without assuming any convexity conditions. Hence, our result is different from many existing ones in the literature (see, e.g., $[6,7,8,25]$ ). Moreover, by weakening the triangle inequality condition, Theorem 4.1 also improves the main results obtained in [2, 3, 4].

Example 4.3. (a) Let $\mathbb{X}=\mathbb{R}^{2}, \mathbb{Y}=\mathbb{R}^{2}, \mathscr{X}=\left\{(x, y) \in \mathbb{R}^{2}: 1 \leq x^{2}+y^{2} \leq 4\right\}$, and $\mathscr{C}=\mathbb{R}_{+}^{2}$. We define $f: \mathbb{R}^{2} \times \mathbb{R}^{2} \rightarrow \mathbb{R}^{2}$ as follows

$$
f(\boldsymbol{x}, \boldsymbol{z})=(\|\boldsymbol{x}-\boldsymbol{a}\|-\|z-\boldsymbol{a}\|,\|z\|-\|\boldsymbol{x}\|),
$$

where $\boldsymbol{a}=(2,0)$. It is clear that $\mathscr{X}$ is compact, and $f$ is $\mathscr{C}$-continuous on $\mathscr{X}$. Now we check that $f$ holds the $\mathscr{C}$-pseudo triangle inequality property on $\mathscr{X}$. If $f(\boldsymbol{y}, \boldsymbol{z}) \preccurlyeq$ int $\mathscr{C} 0$, that is, $(\|y-a\|-\|z-a\|,\|z\|-\|y\|) \in-\operatorname{int} \mathscr{C}$, then

$$
\|\boldsymbol{y}-\boldsymbol{a}\|<\|z-\boldsymbol{a}\| \text { and }\|z\|<\|\boldsymbol{y}\| \text {. }
$$

Therefore, for all $\boldsymbol{x} \in \mathscr{X}$,

$$
\|x-a\|-\|z-a\|<\|x-a\|-\|y-a\| \text { and }\|z\|-\|x\|<\|y\|-\|x\|,
$$


or equivalently $f(\boldsymbol{x}, \boldsymbol{z}) \preccurlyeq$ int $\mathscr{C} f(\boldsymbol{x}, \boldsymbol{y})$. Hence, all the assumptions of Theorem 4.1 are fulfilled. So, we conclude that $\operatorname{WEff}(\mathscr{X}, f)$ is nonempty and compact. By direct computation,

$$
\operatorname{WEff}(\mathscr{X}, f)=\left\{(x, y) \in \mathbb{R}^{2}: x^{2}+y^{2}=1\right\} \cup\left\{(x, 0) \in \mathbb{R}^{2}:-2 \leq x \leq-1\right\} .
$$

(b) Let $\mathbb{X}=\mathbb{R}, \mathbb{Y}=\mathbb{R}^{2}, \mathscr{X}=[-1,1]$, and $\mathscr{C}=\mathbb{R}_{+}^{2}$. The vector bifunction $f: \mathbb{R} \times \mathbb{R} \rightarrow \mathbb{R}^{2}$ is defined by

$$
f(x, z):=\left((z-x)^{3}, z-x\right), \quad \forall x, z \in \mathbb{R} .
$$

Then, $\mathscr{X}$ is compact and $f$ is continuous on $\mathscr{X}$. We now show that $f$ satisfies the int $\mathbb{R}_{+}^{2}$ pseudo triangle inequality condition on $\mathscr{X}$. If $f(y, z) \preccurlyeq \operatorname{int} \mathscr{C} 0$, namely

$$
\left((z-y)^{3}, z-y\right) \in-\operatorname{int} \mathbb{R}_{+}^{2},
$$

then we achieve $z<y, z-x<y-x$, and consequently

$$
f(x, z) \preccurlyeq_{\operatorname{int} \mathscr{C}} f(x, y), \quad \forall x \in \mathscr{X} .
$$

Thus, the assumptions of Theorem 4.1 hold true, and so $\operatorname{WEff}(\mathscr{X}, f)$ is nonempty and compact (in the fact that $\operatorname{WEff}(\mathscr{X}, f)=\{-1\}$ ). However, $f$ is neither concave in the first component nor convex in the second component on $\mathscr{X}$, the results of $[6,7,8,25]$ do not work. Furthermore, $f$ does not hold the triangle inequality condition defined by (4.1), due to

$$
f(1,0)=(-1,-1) \aleph_{\mathscr{C}}(-7,-1)=f(1,2)+f(2,0) .
$$

Hence, the results given in $[2,3,4]$ also cannot be applied.

In order to study the connectedness properties of sets, we employ the following important result.

Lemma 4.1. [43, Proposition 3.1.8, page 81] Assume that $\mathscr{X}$ is a connected subset of $\mathbb{X}$, and a set-valued mapping $W: \mathbb{X} \rightrightarrows \mathbb{Y}$ is upper semicontinuous with connected values on $\mathscr{X}$. Then, $W(\mathscr{X})$ is connected.

Next, we formulate sufficient conditions for the connectedness of $\operatorname{WEff}(\mathscr{X}, f)$ via the nonlinear scalarization function $\eta$.

Theorem 4.2. Let $\mathscr{X}$ be a connected and compact subset of $\mathbb{X}$. Assume that

(i) $f$ is $\mathscr{C}$-continuous and equilibrium on $\mathscr{X} \times \mathscr{X}$, i.e., $f(x, x)=0$ for all $x \in \mathscr{X}$;

(ii) $f$ is int $\mathscr{C}$-pseudo triangle inequality property on $\mathscr{X}$;

(iii) $f$ is naturally quasi connected $\mathscr{C}$-convex in the second component on $\mathscr{X}$.

Then, $\operatorname{WEff}(\mathscr{X}, f)$ is nonempty and connected.

Proof. The proof is divided into four steps.

Step 1. We prove that

$$
\operatorname{WEff}(\mathscr{X}, f)=\bigcup_{x \in \mathscr{X}} W(x),
$$

where $W: \mathscr{X} \rightrightarrows \mathscr{X}$ is defined by $W(x):=\{\bar{z} \in \mathscr{X}:$ for all $z \in \mathscr{X}, \eta(x, z) \geq \eta(x, \bar{z})\}$ for all $x \in \mathscr{X}$. It follows from the proof of Theorem 4.1 that $\operatorname{WEff}(\mathscr{X}, f)$ is nonempty and

$$
\operatorname{WEff}(\mathscr{X}, f) \supset \bigcup_{x \in \mathscr{X}} W(x) \text {. }
$$


Conversely, let $\bar{x} \in \operatorname{WEff}(\mathscr{X}, f)$ be arbitrary. Then

$$
f(\bar{x}, z) \notin-\operatorname{int} \mathscr{C}, \quad \forall z \in \mathscr{X} .
$$

Because of (i), one has $f(\bar{x}, \bar{x})=0$, and hence

$$
\eta(\bar{x}, \bar{x})=\hbar_{-\mathscr{C}}(f(\bar{x}, \bar{x}))=0 .
$$

Applying (4.5), (4.6), and Lemma 2.2(b), we obtain

$$
\eta(\bar{x}, z)=\hbar_{-\mathscr{C}}(f(\bar{x}, z)) \geq 0=\eta(\bar{x}, \bar{x}),
$$

and consequently $\bar{x} \in W(\bar{x}) \subset \bigcup_{x \in \mathscr{X}} W(x)$. Therefore, statement (4.4) holds true.

Step 2. We claim that the set-valued mapping $W$ is upper semicontinuous on $\mathscr{X}$.

Suppose on the contrary that there exists an element $\hat{x}$ of $\mathscr{X}$ such that $W$ is not usc at $\hat{x}$. Then, we can find an open neighborhood $\mathscr{U}$ of $W(\hat{x})$ and a sequence $\left\{\hat{x}_{n}\right\}$ converging to $\hat{x}$ such that, for each $n$, there is $\hat{z}_{n} \in W\left(\hat{x}_{n}\right) \backslash \mathscr{U}$. By the compactness of $\mathscr{X}$, we assume that the sequence $\left\{\hat{z}_{n}\right\}$ converges to some vector $\hat{z}$ belonging to $\mathscr{X}$. If $\hat{z} \notin W(\hat{x})$, then there is $\tilde{z} \in \mathscr{X}$ such that

$$
\eta(\hat{x}, \tilde{z})<\eta(\hat{x}, \hat{z}) \text {. }
$$

Since $\hat{z}_{n} \in W\left(\hat{x}_{n}\right)$, we have $\eta\left(\hat{x}_{n}, \tilde{z}\right) \geq \eta\left(\hat{x}_{n}, \hat{z}_{n}\right)$. This together with the $\mathscr{C}$-continuity of $f$ and Lemma 2.3(c) would imply that $\eta(\hat{x}, \tilde{z}) \geq \eta(\hat{x}, \hat{z})$, which contradicts (4.7). Thus, $\hat{z}$ belongs to $W(\hat{x})$, which is absurd as $\hat{z}_{n} \notin \mathscr{U}$ for all $n$, and hence $W$ is usc on $\mathscr{X}$.

Step 3. For each $x \in \mathscr{X}, W(x)$ is connected.

For each $x \in \mathscr{X}$, let $z_{1}, z_{2}$ be arbitrary in $W(x)$. Then

$$
\eta\left(x, z_{1}\right)=\eta\left(x, z_{2}\right) \text { and } \eta\left(x, z_{i}\right) \leq \eta(x, z), \quad \forall z \in \mathscr{X}, \forall i \in\{1,2\} .
$$

Because of the naturally quasi connected $\mathscr{C}$-convexity of $f$, there exists a connected set $\mathscr{K}_{z_{1}, z_{2}} \subset$ $\mathscr{X}$ containing $z_{1}, z_{2}$ such that

$$
\forall z^{*} \in \mathscr{K}_{z_{1}, z_{2}}, \exists s \in[0,1]: f\left(x, z^{*}\right) \preccurlyeq \mathscr{C}(1-s) f\left(x, z_{1}\right)+s f\left(x, z_{2}\right) .
$$

By Lemma 2.2(a),(d), one has

$$
\forall z^{*} \in \mathscr{K}_{z_{1}, z_{2}}, \exists s \in[0,1]: \eta\left(x, z^{*}\right) \leq(1-s) \eta\left(x, z_{1}\right)+s \eta\left(x, z_{2}\right) .
$$

It follows from (4.8) and (4.9) that

$$
\eta\left(x, z^{*}\right) \leq \eta\left(x, z_{1}\right) \leq \eta(x, z), \quad \forall z \in \mathscr{X}, \forall z^{*} \in \mathscr{K}_{z_{1}, z_{2}} .
$$

Consequently, $\mathscr{K}_{z_{1}, z_{2}} \subset W(x)$, and hence $W(x)$ is connected.

Step 4. Finally, based on Steps 2 and 3, the conditions of Lemma 4.1 are satisfied with the set-valued mapping $W$. In view of Step 1, we obtain the conclusion immediately.

Here, we provide an example to illustrate the applicability of Theorem 4.2.

Example 4.4. Let $\mathbb{X}=\mathbb{R}^{2}, \mathbb{Y}=\mathbb{R}^{2}, \mathscr{X}=\left\{(x, y) \in \mathbb{R}^{2}: x^{2}+y^{2} \leq 1\right\}, \mathscr{C}=\mathbb{R}_{+}^{2}$, and $f: \mathbb{R}^{2} \times \mathbb{R}^{2} \rightarrow$ $\mathbb{R}^{2}$ be defined by

$$
f(\boldsymbol{x}, \boldsymbol{z})=\left(\left(z_{1} z_{2}\right)^{2}-\left(x_{1} x_{2}\right)^{2},\|\boldsymbol{z}\|-\|\boldsymbol{x}\|\right), \quad \forall \boldsymbol{x}=\left(x_{1}, x_{2}\right), \boldsymbol{z}=\left(z_{1}, z_{2}\right) \in \mathbb{R}^{2} .
$$

Then, $\mathscr{X}$ is compact, and $f$ is $\mathscr{C}$-continuous and satisfies the $\mathscr{C}$-pseudo triangle inequality property on $\mathscr{X}$. In order to apply Theorem 4.2 , we need only to check the naturally quasi connected $\mathscr{C}$-convexity of $f$. For each $\boldsymbol{x}=\left(x_{1}, x_{2}\right), \overline{\boldsymbol{z}}=\left(\bar{z}_{1}, \bar{z}_{2}\right)$ and $\tilde{\boldsymbol{z}}=\left(\tilde{z}_{1}, \tilde{z}_{2}\right)$ in $\mathscr{X}$, we consider the connected set $\mathscr{K}_{\bar{z}, \tilde{z}}=\mathscr{L}_{\bar{z}, 0} \cup \mathscr{L}_{\mathbf{0}, \tilde{z}}$, where $0=(0,0)$. 
Next, we show that, for each $z \in \mathscr{K}_{\bar{z}, \tilde{z}}$, we can choose $s \in[0,1]$ such that

$$
f(\boldsymbol{x}, \boldsymbol{z}) \preccurlyeq \mathscr{C}(1-s) f(\boldsymbol{x}, \bar{z})+s f(\boldsymbol{x}, \tilde{z}) .
$$

We have two cases.

Case 1. If $z \in \mathscr{L}_{\bar{z}, 0}$, then there exists $\bar{t} \in[0,1]$ such that $z=\bar{t} \bar{z}$. Consequently,

$$
f(\boldsymbol{x}, \boldsymbol{z})=\left(\left(\bar{t}^{2} \bar{z}_{1} \bar{z}_{2}\right)^{2}-\left(x_{1} x_{2}\right)^{2}, \bar{t}\|\overline{\boldsymbol{z}}\|-\|\boldsymbol{x}\|\right) \preccurlyeq \mathscr{C} f(\boldsymbol{x}, \overline{\boldsymbol{z}}),
$$

and hence (4.10) holds with $s=0$.

Case 2. If $z \in \mathscr{L}_{\mathbf{0}, \tilde{z}}$, then we can find $\tilde{t} \in[0,1]$ such that $z=\tilde{t} \tilde{z}$. This leads to

$$
f(\boldsymbol{x}, \boldsymbol{z})=\left(\left(\tilde{t}^{2} \tilde{z}_{1} \tilde{z}_{2}\right)^{2}-\left(x_{1} x_{2}\right)^{2}, \tilde{t}\|\tilde{\boldsymbol{z}}\|-\|\boldsymbol{x}\|\right) \preccurlyeq \mathscr{C} f(\boldsymbol{x}, \tilde{\boldsymbol{z}}),
$$

and consequently (4.10) satisfies with $s=1$.

Therefore, by Theorem 4.2, the set $\operatorname{WEff}(\mathscr{X}, f)$ is nonempty and connected.

Definition 4.3. [44, Definition 2.19, page 113] A vector-valued mapping $g: \mathbb{X} \rightarrow \mathbb{Y}$ is said to be $\mathscr{C}$-downward directed on $\mathscr{X}$ if, for any $x_{1}, x_{2} \in \mathscr{X}$, there exists $\bar{x} \in \mathscr{X}$ such that

$$
g(\bar{x}) \preccurlyeq \mathscr{C} g\left(x_{1}\right) \text { and } g(\bar{x}) \preccurlyeq \mathscr{C} g\left(x_{2}\right) \text {. }
$$

Now, we use the triangle inequality together with the downward directedness property of $f$ to establish the existence conditions of strongly efficient solution sets of nonconvex vector equilibrium problems by the direct approach.

Theorem 4.3. Let $\mathscr{X}$ be compact. Assume that

(i) $f$ holds the triangle inequality property on $\mathscr{X}$;

(ii) there exists some vector $\bar{x} \in \mathscr{X}$ such that $f(\bar{x}, \cdot)$ is $\mathscr{C}$-downward directed and $\mathscr{C}$-lower pseudocontinuous on $\mathscr{X}$.

Then, $\operatorname{SEff}(\mathscr{X}, f)$ is nonempty.

Proof. Let $\bar{x}$ be given by (ii). For each $z \in \mathscr{X}$, we consider the following set

$$
\mathscr{L}(\bar{x}, z):=\{\bar{z} \in \mathscr{X}: f(\bar{x}, \bar{z}) \preccurlyeq \mathscr{C} f(\bar{x}, z)\} .
$$

Then, $z \in \mathscr{L}(\bar{x}, z)$, and so $\mathscr{L}(\bar{x}, z)$ is nonempty. Since $\mathscr{X}$ is closed and $f(\bar{x}, \cdot)$ is $\mathscr{C}$-lower pseudocontinuous on $\mathscr{X}$, the set $\mathscr{L}(\bar{x}, z)=\operatorname{lev}_{\preccurlyeq f(\bar{x}, z)} f(\bar{x}, \cdot) \cap \mathscr{X}$ is closed. We show that

$$
\bigcap_{z \in \mathscr{X}} \mathscr{L}(\bar{x}, z) \neq \emptyset \text {. }
$$

Let $\left\{z_{1}, z_{2}, \cdots, z_{n}\right\}$ be a finite subset of $\mathscr{X}$. Since $f(\bar{x}, \cdot)$ is downward directed, there is $z_{0} \in \mathscr{X}$ such that $f\left(\bar{x}, z_{0}\right) \preccurlyeq \mathscr{C} f\left(\bar{x}, z_{i}\right), \forall i \in \mathscr{I}:=\{1,2, \cdots, n\}$. Consequently, $z_{0} \in \mathscr{L}\left(\bar{x}, z_{i}\right)$ for all $i \in \mathscr{I}$, and $\cap_{i \in \mathscr{I}} \mathscr{L}\left(\bar{x}, z_{i}\right) \neq \emptyset$, for any finite subset $\mathscr{I}$ of $\mathscr{X}$. Because $\mathscr{X}$ is compact, and for each $x \in \mathscr{X}, \mathscr{L}(\bar{x}, z)$ is a closed subset of $\mathscr{X}$, statement (4.11) will follow from Lemma 2.1. For every $x_{0} \in \bigcap_{z \in \mathscr{X}} \mathscr{L}(\bar{x}, z)$, we have

$$
f\left(\bar{x}, x_{0}\right) \preccurlyeq \mathscr{C} f(\bar{x}, z), \quad \forall z \in \mathscr{X} .
$$

Combining (4.12) with the triangle inequality property of $f$, one has

$$
f(\bar{x}, z) \preccurlyeq \mathscr{C} f\left(\bar{x}, x_{0}\right)+f\left(x_{0}, z\right) \preccurlyeq \mathscr{C} f(\bar{x}, z)+f\left(x_{0}, z\right),
$$

and consequently $0 \preccurlyeq \mathscr{C} f\left(x_{0}, z\right)$ for all $z \in \mathscr{X}$. Hence, $x_{0}$ is a strongly efficient solution of (VEP). The proof is complete. 
Example 4.5. Let $\mathbb{X}=\mathbb{R}, \mathbb{Y}=\mathbb{R}^{2}, \mathscr{X}=[-1,1]$, and $\mathscr{C}=\mathbb{R}_{+}^{2}$. The vector bifunction $f: \mathbb{R} \times$ $\mathbb{R} \rightarrow \mathbb{R}^{2}$ is defined by

$$
f(x, z):=\left(z-x, z^{3}-x^{3}\right), \quad \forall x, z \in \mathbb{R} .
$$

Then, $\mathscr{X}$ is compact and $f$ is continuous on $\mathscr{X}$. Moreover, for all $x, y, z \in \mathbb{R}$, we have

$$
\begin{aligned}
f(x, z) & =\left(z-x, z^{3}-x^{3}\right)=\left(y-x, y^{3}-x^{3}\right)+\left(z-y, z^{3}-y^{3}\right) \\
& \preccurlyeq \mathscr{C} f(x, y)+f(y, z),
\end{aligned}
$$

and so $f$ satisfies the triangle inequality property on $\mathscr{X}$. Next, let $\bar{x}=0$. Then, for any $z_{1}, z_{2} \in$ $[-1,1]$, by choosing $\bar{z}=-1$, we have

$$
f(0, \bar{z}) \preccurlyeq \mathscr{C} f\left(0, z_{1}\right) \text { and } f(0, \bar{z}) \preccurlyeq \mathscr{C} f\left(0, z_{2}\right) \text {, }
$$

and so $f(0, \cdot)$ is $\mathscr{C}$-downward directed. Therefore, all the assumptions of Theorem 4.3 are satisfied, and thus the set $\operatorname{SEff}(\mathscr{X}, f)$ is nonempty (in fact, $\operatorname{SEff}(\mathscr{X}, f)=\{-1\})$.

Theorem 4.4. Let $\mathscr{X}$ be a connected and compact subset of $\mathbb{X}$. Assume that

(i) $f$ is $\mathscr{C}$-continuous and equilibrium on $\mathscr{X} \times \mathscr{X}$;

(ii) $f$ satisfies the triangle inequality property on $\mathscr{X}$;

(iii) $f$ is $\mathscr{C}$-downward directed as well as naturally quasi connected $\mathscr{C}$-convex in the second component on $\mathscr{X}$.

Then, $\operatorname{SEff}(\mathscr{X}, f)$ is nonempty and connected.

Proof. In view of Theorem 4.3 , the set $\operatorname{SEff}(\mathscr{X}, f)$ is nonempty. Let $S: \mathscr{X} \rightrightarrows \mathscr{X}$ be a setvalued mapping defined by

$$
S(x)=\bigcap_{z \in \mathscr{X}} \mathscr{L}(x, z), \quad \forall x \in \mathscr{X},
$$

where $\mathscr{L}(\bar{x}, z):=\{\bar{z} \in \mathscr{X}: f(\bar{x}, \bar{z}) \preccurlyeq \mathscr{C} f(\bar{x}, z)\}$. Then, for all $x \in \mathscr{X}$,

$$
S(x)=\{\bar{z} \in \mathscr{X}: \text { for all } z \in \mathscr{X}, f(x, \bar{z}) \preccurlyeq \mathscr{C} f(x, z)\} .
$$

Similar to Theorem 4.2, we also consider the following steps.

Step 1. $\operatorname{SEff}(\mathscr{X}, f)=\bigcup_{x \in \mathscr{X}} S(x)$ :

By Theorem 4.3, for each $x \in \mathscr{X}, S(x)$ is a nonempty subset of $\operatorname{SEff}(\mathscr{X}, f)$, and hence

$$
\bigcup_{x \in \mathscr{X}} S(x) \subset \operatorname{SEff}(\mathscr{X}, f) \text {. }
$$

Conversely, for any $x \in \operatorname{SEff}(\mathscr{X}, f)$, due to (i), one has $f(x, x)=0 \preccurlyeq \mathscr{C} f(x, z), \forall z \in \mathscr{X}$, and consequently $x \in S(x)$. So,

$$
\operatorname{SEff}(\mathscr{X}, f) \subset \bigcup_{x \in \mathscr{X}} S(x) .
$$

Step 2. For each $x \in \mathscr{X}, S(x)$ is connected:

Let $z_{1}, z_{2} \in S(x)$ be arbitrary. Then, for all $z \in \mathscr{X}$,

$$
f\left(x, z_{1}\right) \preccurlyeq \mathscr{C} f(x, z) \text { and } f\left(x, z_{2}\right) \preccurlyeq \mathscr{C} f(x, z) .
$$

On the other hand, since $f(x, \cdot)$ is naturally quasi connected $\mathscr{C}$-convex on $\mathscr{X}$, there exists a connected set $\mathscr{K}_{z_{1}, z_{2}} \subset \mathscr{X}$ containing $z_{1}, z_{2}$ such that, for each $\bar{z} \in \mathscr{K}_{z_{1}, z_{2}}$, we can find $s \in[0,1]$, $f(x, \bar{z}) \preccurlyeq \mathscr{C}(1-s) f\left(x, z_{1}\right)+s f\left(x, z_{2}\right)$. Combining this with (4.13), we obtain

$$
f(x, \bar{z}) \preccurlyeq \mathscr{C}(1-s) f(x, z)+s f(x, z)=f(x, z), \quad \forall z \in \mathscr{X} .
$$


Equivalently, $\bar{z} \in S(x)$, and so $\mathscr{K}_{z_{1}, z_{2}} \subset S(x)$. Therefore, $S(x)$ is connected.

Step 3. The set-valued mapping $S$ is upper semicontinous on $\mathscr{X}$ :

Suppose on the contrary that we can find some vector $\hat{x} \in \mathscr{X}$ such that $S$ is not usc at $\hat{x}$. Then, there are an open neighborhood $\mathscr{U}$ of $S(\hat{x})$ and a sequence $\left\{\hat{x}_{n}\right\}$ converging to $\hat{x}$ such that, for each $n$, there exists $\hat{z}_{n} \in S\left(\hat{x}_{n}\right) \backslash \mathscr{U}$. Since $\mathscr{X}$ is compact, we can assume that $\left\{\hat{z}_{n}\right\}$ converges to some vector $\hat{z} \in \mathscr{X}$. If $\hat{z} \notin S(\hat{x})$, then there is $\tilde{z} \in \mathscr{X}$ such that

$$
f(\hat{x}, \hat{z}) \npreceq \mathscr{C} f(\hat{x}, \tilde{z}) .
$$

Due to $\hat{z}_{n} \in S\left(\hat{x}_{n}\right)$, we have

$$
f\left(\hat{x}_{n}, \hat{z}_{n}\right) \preccurlyeq \mathscr{C} f\left(\hat{x}_{n}, \tilde{z}\right) .
$$

Because $f$ is $\mathscr{C}$-continuous, (4.15) together with [45, Propositions 2.3, 2.4] would imply that $f(\hat{x}, \hat{z}) \preccurlyeq \mathscr{C} f(\hat{x}, \tilde{z})$, which contradicts (4.14). So, $\hat{z} \in S(\hat{x}) \subset \mathscr{U}$. It is impossible as $\hat{z}_{n} \notin \mathscr{U}$ for all $n$.

Step 4. Employing Lemma 4.1 and $\operatorname{Steps} 1-3$, we conclude that $\operatorname{SEff}(\mathscr{X}, f)$ is connected.

Remark 4.2. In Theorems 4.2 and 4.4, we obtain connectedness conditions for weakly and strongly efficient solution sets of (VEP) without assuming convexity and monotonicity of the constraint sets and the objective mappings. Hence, our approaches and results are new and different from the existing ones in the literature; see, e.g., [22, 24, 25, 28, 29] and the references therein.

\section{Acknowledgments}

The authors would like to thank anonymous referees for their valuable remarks and suggestions which helped us to improve the paper. This is a result of the project under Grant number B2022TCT-02, supported by The Ministry of Education and Training of Viet Nam.

\section{REFERENCES}

[1] G. Kassay, V. Rădulescu, Equilibrium Problems and Applications, Academic Press, London, 2018.

[2] S. László, Vector equilibrium problems on dense sets, J. Optim. Theory Appl. 170 (2016), 437-457.

[3] M. Bianchi, G. Kassay, R. Pini, Ekeland's principle for vector equilibrium problems, Nonlinear Anal. 66 (2007), 1454-1464.

[4] G. Xunhua, Ekeland's principle for set-valued vector equilibrium problems, Acta Math. Sci. 34 (2014), 11791192.

[5] Q.H. Ansari, J.C. Yao, An existence result for the generalized vector equilibrium problem, Appl. Math. Lett. 12 (1999), 53-56.

[6] Q.H. Ansari, A.P. Farajzadeh, S. Schaible, Existence of solutions of strong vector equilibrium problems, Taiwanese J. Math. 16 (2012), 165-178.

[7] A.P. Farajzadeh, R. Wangkeeree, J. Kerdkaew, On the existence of solutions of symmetric vector equilibrium problems via nonlinear scalarization, Bull. Iranian Math. Soc. 45 (2019), 35-58.

[8] G. Bigi, A. Capătă, G. Kassay, Existence results for strong vector equilibrium problems and their applications, Optimization 61 (2012), 567-583.

[9] L.Q. Anh, P.T. Duoc, T.N. Tam, N.C. Thang, Stability analysis for set-valued equilibrium problems with applications to browder variational inclusions, Optim. Lett. 15 (2021), 613-626.

[10] L.Q. Anh, P.T. Duoc, T.N. Tam, On the stability of approximate solutions to set-valued equilibrium problems, Optimization 69 (2020), 1583-1599.

[11] L.Q. Anh, P.T. Duoc, T.N. Tam, On Hölder continuity of solution maps to parametric vector primal and dual equilibrium problems, Optimization 67 (2018), 1169-1182. 
[12] Y. Han, X.H. Gong, Lower semicontinuity of solution mapping to parametric generalized strong vector equilibrium problems, Appl. Math. Lett. 28 (2014), 38-41.

[13] Z.Y. Peng, X.J. Long, X.F. Wang, Y.B. Zhao, Generalized hadamard well-posedness for infinite vector optimization problems, Optimization 66 (2017), 1563-1575.

[14] I. Sadeqi, M. Salehi Paydar, Lipschitz continuity of an approximate solution mapping for parametric setvalued vector equilibrium problems, Optimization 65 (2016), 1003-1021.

[15] S. Xu, S.J. Li, A new proof approach to lower semicontinuity for parametric vector equilibrium problems, Optim. Lett. 3 (2009), 453-459.

[16] Y.D. Xu, C.R. Chen, C.J. Fang, Hölder continuity for solution mappings of parametric non-convex strong generalized Ky Fan inequalities, Numer. Funct. Anal. Optim. 41 (2020), 344-360.

[17] O. Chadli, Q.H. Ansari, S. Al-Homidan, Existence of solutions and algorithms for bilevel vector equilibrium problems: an auxiliary principle technique, J. Optim. Theory Appl. 172 (2017), 726-758.

[18] S.H. Wang, Q.Y. Li, A projection iterative algorithm for strong vector equilibrium problem, Optimization 64 (2015), 2049-2063.

[19] A.N. Iusem, V. Mohebbi, Extragradient methods for vector equilibrium problems in Banach spaces, Numer. Funct. Anal. Optim. 40 (2019), 993-1022.

[20] S.H. Wang, J.H. Huang, C.X. Zhu, An iterative method for solving the strong vector equilibrium problem with variable domination structure, Optimization 67(2018), 865-879.

[21] Q.H. Ansari, F. Flores-Bazán, Recession methods for generalized vector equilibrium problems, J. Math. Anal. Appl. 321 (2006), 132-146.

[22] X.H. Gong, Efficiency and Henig efficiency for vector equilibrium problems, J. Optim. Theory Appl. 108 (2001), 139-154.

[23] X.H. Gong, Connectedness of the solution sets and scalarization for vector equilibrium problems, J. Optim. Theory Appl. 133 (2007), 151-161.

[24] X.H. Gong, J.C. Yao, Connectedness of the set of efficient solutions for generalized systems, J. Optim. Theory Appl. 138 (2008), 189-196

[25] Y. Han, N.J. Huang, Existence and connectedness of solutions for generalized vector quasi-equilibrium problems, J. Optim. Theory Appl. 179 (2018), 65-85.

[26] Z. Peng, Z. Wang, X. Yang, Connectedness of solution sets for weak generalized symmetric Ky Fan inequality problems via addition-invariant sets, J. Optim. Theory Appl. 185 (2020), 188-206.

[27] P.H. Sach, Connectedness in vector equilibrium problems involving cones with possibly empty interior, Oper. Res. Lett. 44 (2016), 177-179.

[28] Y. Xu, P. Zhang, Connectedness of solution sets of strong vector equilibrium problems with an application, J. Optim. Theory Appl. 178 (2018), 131-152.

[29] C.Y. Shao, Z.Y. Peng, Y.B. Xiao, Y. Zhao, Connectedness of solution sets for generalized vector equilibrium problems via free-disposal sets in complete metric space, Optim. Lett. 172 (2021), 1-21.

[30] A. Göpfert, H. Riahi, C. Tammer, C. Zalinéscu, Variational Methods in Partially Ordered Spaces, Springer, Berlin, 2003.

[31] D.T. Luc, Theory of Vector Optimization. Lecture Notes in Economics and Mathematical Systems, vol. 319, Springer, Berlin, 1989.

[32] L.Q. Anh, T.Q. Duy, D.V. Hien, Stability for parametric vector quasi-equilibrium problems with variable cones, Numer. Funct. Anal. Optim. 40 (2019), 461-483.

[33] N. Bourbaki, General Topology: Chapters 1-4, vol. 18, Springer, Berlin, 1987.

[34] J. Morgan, V. Scalzo, Pseudocontinuity in optimization and nonzero sum games, J. Optim. Theory Appl. 120 (2004), 181-197.

[35] W.J. Pervin, Foundations of General Topology, Academic Press, London, 1964.

[36] J.B. Hiriart-Urruty, Tangent cones, generalized gradients and mathematical programming in Banach spaces, Math. Oper. Res. 4 (1979), 79-97.

[37] B. Jiménez, V. Novo, A. Vílchez, Characterization of set relations through extensions of the oriented distance, Math. Methods Oper. Res. 91 (2020), 89-115.

[38] R.T. Rockafellar, Convex Analysis, Princeton University Press, Princeton, NJ, 1970. 
[39] M. Avriel, I. Zang, Generalized arcwise-connected functions and characterizations of local-global minimum properties, J. Optim. Theory Appl. 32 (1980), 407-425.

[40] A.R. Warburton, Quasiconcave vector maximization: connectedness of the sets of pareto-optimal and weak pareto-optimal alternatives, J. Optim. Theory Appl. 40 (1983), 537-557.

[41] T. Tanaka, Generalized quasiconvexities, cone saddle points, and minimax theorem for vector-valued functions, J. Optim. Theory Appl. 81 (1994), 355-377.

[42] D. Monderer, L.S. Shapley, Potential games, Games Econ. Behav. 14 (1996), 124-143.

[43] A.A. Khan, C. Tammer, C. Zalinéscu, Set-Valued Optimization, Springer, Berlin, 2016.

[44] Q.H. Ansari, E. Köbis, J.C. Yao, Vector Variational Inequalities and Vector Optimization, Springer, Berlin, 2018.

[45] L.Q. Anh, P.Q. Khanh, D.T.M. Van, J.C. Yao, Well-posedness for vector quasiequilibria, Taiwanese J. Math. 13 (2009), 713-737. 